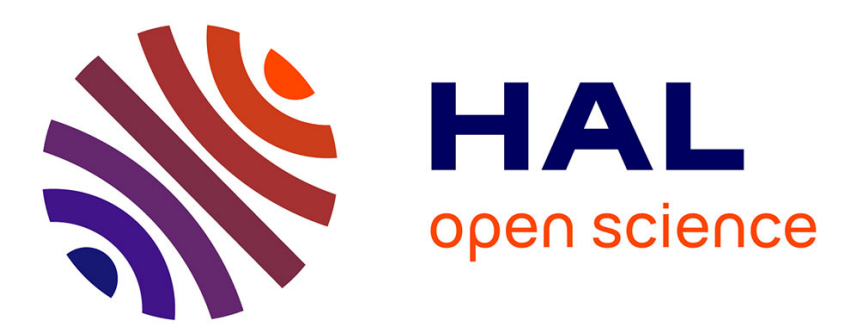

\title{
Imaging the interior of a comet from bistatic microwave measurements: case of a scale comet model
}

Christelle Eyraud, Alain Hérique, Jean-Michel Geffrin, Wlodek W. Kofman

\section{To cite this version:}

Christelle Eyraud, Alain Hérique, Jean-Michel Geffrin, Wlodek W. Kofman. Imaging the interior of a comet from bistatic microwave measurements: case of a scale comet model. Advances in Space Research, 2018, 62 (8), pp.1977-1986. 10.1016/j.asr.2017.10.012 . hal-01626737

\section{HAL Id: hal-01626737 https://hal.science/hal-01626737}

Submitted on 31 Oct 2018

HAL is a multi-disciplinary open access archive for the deposit and dissemination of scientific research documents, whether they are published or not. The documents may come from teaching and research institutions in France or abroad, or from public or private research centers.
L'archive ouverte pluridisciplinaire HAL, est destinée au dépôt et à la diffusion de documents scientifiques de niveau recherche, publiés ou non, émanant des établissements d'enseignement et de recherche français ou étrangers, des laboratoires publics ou privés. 


\title{
Imaging the interior of a comet from bistatic microwave measurements: case of a scale comet model
}

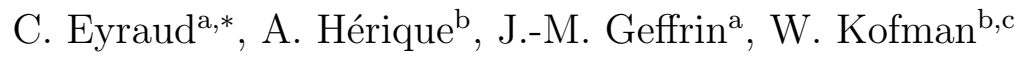 \\ ${ }^{a}$ Aix Marseille Univ, CNRS, Centrale Marseille, Institut Fresnel, F-13013 Marseille, \\ France \\ ${ }^{b}$ Univ. Grenoble Alpes, CNRS, IPAG, F-38000 Grenoble, France \\ ${ }^{c}$ Space Research Centre, PAS, 00-001 Warsaw, Poland
}

\begin{abstract}
Imaging the internal structure of comets and asteroids is an important way to provide information about their formation process. In this paper, we investigate the possibility to image the interior of such structures with electromagnetic wave in the microwave domain (radar system) using an inverse algorithm adapted to take advantage of a bistatic configuration, taking into account the polarization effects, and which presents low memory requirement. To this end, a scale model of a comet/asteroid was built and was used for an experimental simulation. The scattered fields of this scale model were measured in a perfectly controlled environment, in an anechoic chamber, to avoid measurement disturbances and to focus this study only on which structural information can be obtained with such measurements. To profit from the spatial diversity of information, a vectorial-induced current reconstruction algorithm was used. Two configurations were tested and analyzed including one with very few measurements. From the qualitative reconstructed maps,
\end{abstract}

\footnotetext{
${ }^{*}$ Corresponding author

Email addresses: christelle.eyraud@fresnel.fr (C. Eyraud)
} 
we have shown that it is possible to detect the presence of a core in both cases.

Key words: Comet analog; Radar Tomography; Imaging; Internal

structure; Bistatic configuration; Electromagnetic wave; Microwave measurements

\section{Introduction}

The interior of comets and asteroids, i.e. their structure and composition, is poorly known, but could provide information to understand the early development of the Solar System (Bottke-Jr. et al. 2002), (Festou et al. 2004). Nethertheless, it is a very challenging problem obviously in terms of measurements but also in terms of imaging procedures. To solve such inverse problem, the difficulties are mainly due to the two following problems: such structures are very large and furthermore the number of measurements in a realistic space radar scenario is necessarily very limited.

The appeal of the sounding of these small solar system bodies with electromagnetic waves has been increasing in recent years (Herique et al. n.d.). The CONSERT experiment on the European Space Agency's Rosetta mission is the first instrument designed to reconstruct the interior of a comet. It met the Comet 67P/Churyumov-Gerasimenko in November 2014. The CONSERT experiment has explored the nucleus of this comet using electromagnetic waves in the radiowave regime and exploiting a bistatic configuration (Kofman et al. 2007). One of its scientific aims is to contribute to a better understanding of the composition of the cometary core and of its internal structure (Kofman et al. 1998), (Kofman \& Safaeinili 2004). This 
experiment is based on a transmission travel-time tomography process working with a central frequency of $90 \mathrm{MHz}$ and a bandwidth of $10 \mathrm{MHz}$. The electromagnetic waves sent by the source on the orbiter Rosetta, propagate inside the comet up to the lander Philae and then are sent back to the orbiter. This experiment takes profit from the rotation of the comet itself to reach several bistatic angles. The first measurements with CONSERT were made immediately after the landing of Philae on the comet (Kofman et al. 2015) and have already shown the ability of such electromagnetic techniques to explore the comets (Kofman et al. 2007), (Kofman et al. 2015), (Herique et al. 2017).

Modelling the propagation of the electromagnetic signal through such large bodies is not trivial as numerical rigorous models based on the discretization of the Maxwell equations are requiring an extremely high memory capacity. To this end, methods based on Physical Optics and the ray-tracing method have been applied to this problem (Kofman et al. 1998) Virkki \& Muinonen 2016), (Hegler et al. 2013), (Ciarletti et al. 2015). To image the interior of the structure, radar techniques which fully exploit the bandwidth (or temporal methods) have been developed. Indeed, the temporal echoes due to the interaction of the electromagnetic waves with the different inhomogeneities were detected. This can be seen with a direct temporal visualization of the signals versus time as radargrams or as B-scan images Asphaug et al. 2010); focusing by migrations methods have also be adapted to process these data (Herique et al. 1999), (Barriot et al. 1999), (Sava et al. 2015), (Grimm et al. 2015).

With such bodies, the non-linear imaging procedures - here named quan- 
titative inversion - which allow to get the complex permittivity maps of the targets are very difficult to exploit, specially for the 3D case. Numerical studies on the imaging of the interior of a body which are based on wave propagation have been conducted in both 2D (Pursiainen \& Kaasalainen 2014) and in 3D geometries (Pursiainen \& Kaasalainen 2015). It is true, however, that 3D inversion for a full wave is still a challenge as the objects are very large compared to the wavelengths, i.e., the number of unknowns is - in the general case - also very large which leads to a huge memory requirement, but also to a high risk of false solution (local minimum solution). This risk is also increased by the small quantity of measurements. For more details on these methods the special section of the Inverse Problems journal devoted to the test of such non-linear inversion algorithms against experimental data on 3D targets can be seen (Litman \& Crocco 2009), Geffrin \& Sabouroux 2009).

First-order diffraction tomography algorithms can be a good solution to reach the structural characteristics of these bodies (and also the electromagnetic characteristics when low-contrasted targets are considered). In these algorithms, the polarizations of the scattered field and the incident field are colinear and the Born or the Rytov approximations are used to formulate this problem with a scalar equation. The contrast map is then retrieved via an inverse Fourier transform (see for example (Gurg \& Wolf 2001), (Safaeinili et al. 2002), (Devaney 2012)).

The present study continues with the same spirit, but we fully take into account the entire vectorial nature of the field which is needed for such tridimensional bodies. Furthermore, no linear assumption is made here to retrieve 
the structural characteristics of the target. The spatial diversity of the information which can be obtained in bistatic scenarios is exploited. This study is conducted on a 3D scale model of a comet, from lab-measurements using a single frequency to reconstruct the images.

The details of the inner structure of this target are explained in Part II. Part III is devoted to the lab-experimentations. Two configurations of measurement are investigated and explained. The information content in these configurations is then analyzed in Part IV. Experimentally measured scattered fields are used as input data of the imaging procedure which is described in Part V. The imaging results are presented and discussed in Part VI. Some concluding remarks follow in part VII.

\section{Scale model of a comet}

Comets can present a wide variety and a diverse set of morphologies has been proposed for the cometary nuclei such as the fractal aggregate model (Donn 1990), rubble-pile model (Weissman 1986), icy glue model (Gombosi \& Houpis 1986), layered pile model (Belton et al. 2007), corresponding to different formation scenarios (Sunshine et al. 2016), (Davidsson et al. 2016). In this study, we chose to work with a body supposed to have the morphology described by the layered pile model. A scale target, based on this predicted model, was made. This layered pile model has been simplified by considering only three parts: the core, a layer corresponding to the crystalline ice crust and dirty ice, and then the dust mantle. A sketch and a photography of this target are presented in Fig. 1. 


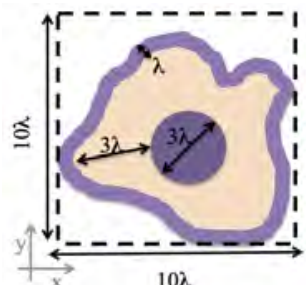

(a)

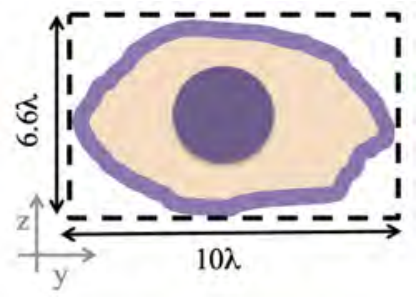

(b)

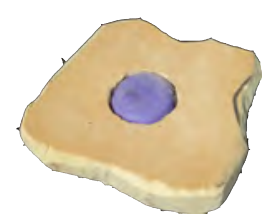

(c)

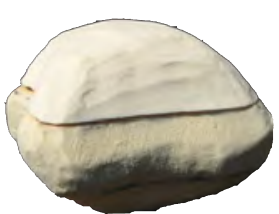

(d)

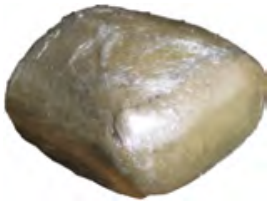

(e)

Figure 1: Comet scale model: (a) sketch in the (x0y) plane, (b) sketch in the (x0z) plane, (c) photography of the interior (in the (x0y) plane), (d) photography of the second layer, (e) photography of the global scale model.

\subsection{Geometrical aspects}

The dimensions of comets are too large in terms of wavelength to allow the calculation of the scattered field of such an object without approximation when using a standard computer. Thus instead of trying to make such heavy computations, we decided to work with measurements performed in a totally controlled environment, i.e., the fields were measured in the anechoic chamber of the Centre Commun de Ressources en Microonde (CCRM) in Marseille.

As such a body is too big for an experimental setup, we used the microwave analogy to reduce the dimensions of the object (Gustafson 2009). Indeed, the microwave analogy takes advantage of the scale invariance of 
the Maxwell equations to rescale both the dimensions of the target and the wavelength by the same factor, keeping the dielectric parameters constant.

As the dimensions of the objet are still too large for our actual setup, we chose to work with a smaller target in this study - a reduced scale model of a comet. The working frequency was chosen to be $18 \mathrm{GHz}$ and the scale model can be included in a sphere of $250 \mathrm{~mm}$-diameter. 67P/CG has a different shape than our scale model, but we can nevertheless compare the size of the minimum sphere enclosing each body: in our scale model, the dimensions have been divided by a scale factor equal to about 90 - in terms of wavelengths. Even if our scale model is smaller, this target can be very useful to understand what kind of information can be retrieved.

The relative size of each layer with respect to the total size of the comet has been respected as much as possible in the building of our model. Indeed, the radial dimensions of the core, of the ice layer and of the dust mantle correspond respectively to $\approx 30 \%, 59 \%$ and $11 \%$ of the total scale model radius.

\subsection{Electromagnetic aspects}

The material of which each layer was made was chosen to keep the same permittivity values as the ones expected in the layer pile model Heggy et al. 2012). It was also of great importance to choose materials with a perfectly known permittivity value. Indeed, for the core and the dust mantle, silica sand was used. Its permittivity was measured with a method based on the bistatic far-field scattering patterns measurements (Eyraud et al. 2015). The silica sand permittivity was found to be equal to $\epsilon_{r}=(2.8 \pm 0.1)+j(0.03 \pm$ $0.04)$ at $18 \mathrm{GHz}$. The ice layer was built with a foam (SAITEC SBF 300) of 
permittivity $\epsilon_{r}=1.45 \pm 0.15$, its permittivity was determined with a coaxial cell method (Sabouroux \& Ba 2011) and then refined with the same method as previously, based on far-field scattering patterns measurements, but using a supposed infinite long cylinder. It is of noting that the permittivity of the comet $67 \mathrm{P} / \mathrm{CG}$ measured by CONSERT is a bit lower and equal to $\epsilon_{r}=1.27 \pm 0.05$ (Kofman et al. 2015), (Herique et al. 2017).

\section{Experiments}

\subsection{Setup}

All the scattered field measurements were made with the equipment of the CCRM in Marseille, which is equipped with a spherical positioning setup inside an anechoic chamber. The working frequencies are in the 1 to $20 \mathrm{GHz}$ range actually, but will be soon upgraded to $40 \mathrm{GHz}$ for scattering measurements (see (Eyraud et al. 2008), (Geffrin et al. 2009) for more details). Using the different positioning devices is equivalent to placing the sources and receivers in a large variety of positions on an approximately 2 meter radius sphere surrounding the target. This object is then assumed to be totally in free space, as it is placed on an expanded polystyrene mast which is totally transparent at those frequencies and as the anechoicity provided by the absorbing materials reduces efficiently the scattering of the surrounding.

The measurements are made in three steps, with (total field) and without (incident field) the target, to extract a raw scattered field through the complex subtraction of those two measured fields. The third step consists in measuring the total field of a known object (a metallic sphere in the present case) to calibrate the measured field (Geffrin et al. 2009). Finally a post- 
processing procedure is applied to remove drift errors and to compensate for the identified residual errors (Eyraud et al. 2006).

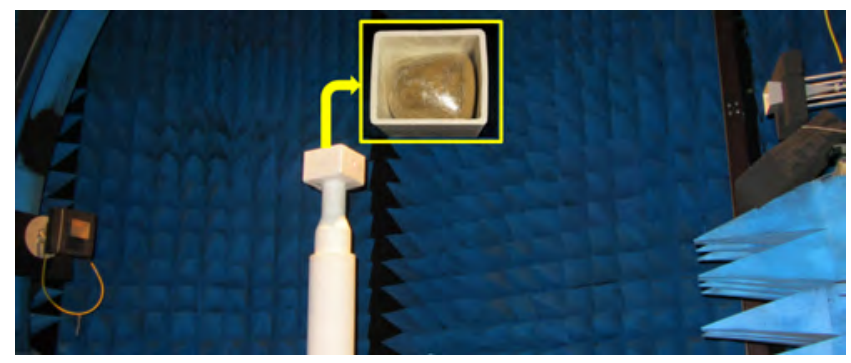

Figure 2: Photography of the anechoic chamber of the CCRM - Marseille.

\subsection{Choice of the configuration}

As in real conditions, very few measurements can be acquired. In this study, we made experiments measuring only a small part of the multistatic scattering matrix. Two configurations were considered, the first one, named $\mathrm{A}$ in the following, with more measurements and the second one, named B, with very few measurement points. The measurements were performed with a single polarization case, i.e., the illumining wave polarized along the $\mathbf{e}_{\phi}^{\mathbf{T}}$ vector and the receiver measuring the electric field along the $\mathbf{e}_{\phi}^{\mathbf{R}}$ vector (see Fig. 3p. It can be noticed that in the general case, the two vectors $\left(\mathbf{e}_{\phi}^{\mathbf{T}}\right.$ and $\left.\mathbf{e}_{\phi}^{\mathbf{R}}\right)$ are not collinear. The measurements were made at a single frequency $(18 \mathrm{GHz}, \lambda=16.7$ ,mm).

\subsubsection{Configuration $A$}

The source of coordinates, $\left(r^{T}, \theta^{T}, \phi^{T}\right)$, was displaced on a sphere of radius $r_{s}=1.691 \mathrm{~m}$ with 13 angular values of the $\phi^{T}$-angle, each one with 18 


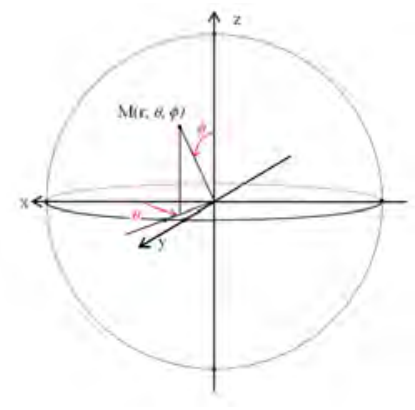

(a)

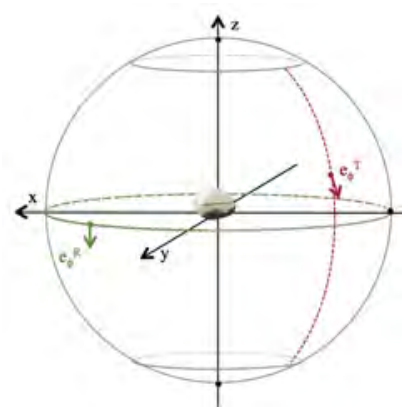

(b)

Figure 3: Sketch of the experimental configuration: (a) definition of the coordinate system, (b) positions of the source (in red), of the receiver (in green) and of the scale model.

angular positions $\theta^{T}$. The receiver was kept in the horizontal $(x 0 y)$ plane and the field was measured with 261 receiver angles (261 values of the $\theta^{R}$-angle equally spaced) with an exclusion zone of $\pm 50^{\circ}$ around the source position due to mechanical contraints (receiver coordinates: $\left(r^{R}, \theta^{R}, \phi^{R}=90^{\circ}\right.$ ) (see Fig. $4(\mathrm{a}))$.

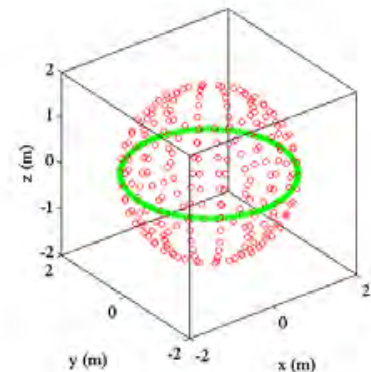

(a) Configuration $\mathrm{A}$

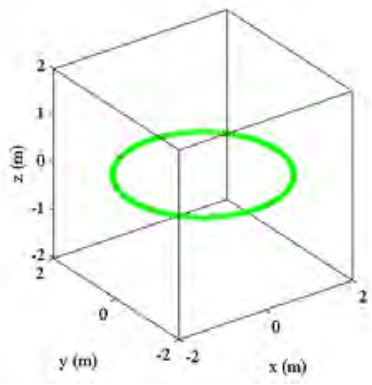

(b) Configuration B

Figure 4: Positions of the transmitters (red circle) and positions of the receivers (green cross) for the two configurations. 


\subsubsection{Configuration $B$}

We chose to keep both the transmitter and the receiver in the same horizontal $(x 0 y)$ plane. Indeed, the coordinates of the transmitting and receiving antenna were, respectively, $\left(r^{T}=1.691 \mathrm{~m}, \theta^{T}, \phi^{T}=90^{\circ}\right)$ and $\left(r^{R}=1.691 m, \theta^{R}, \phi^{R}=90^{\circ}\right) .9$ illuminations were considered (9 values of the $\theta^{T}$ angle equally spaced). The field was measured on 261 receiver angles (261 values for the $\theta^{R}$ angle) equally spaced too with the same exclusion zone of $\pm 50^{\circ}$ around the source position (see Fig. 4 (b)). Notice that even though the objet is a $3 \mathrm{D}$ one, a 2D acquisition configuration is used. Moreover, keep in mind that some of the measurements can be redundant, since the measured field is oversampled (as explained in the next section 4).

\section{Analysis of the information content}

The maximal amount of information in far field that can be obtained for an arbitrary transmitter-receiver configuration follows from the size of the target and the illuminating frequency (Bucci \& Isernia 1997). In the case of our scale model, the number of degrees of freedom of the scattered field is equal to $128 \pi^{2}\left(\frac{a}{\lambda}\right)^{4} \approx 3500000$ where $\lambda$ is the wavelength of the electromagnetic wave and $a$ is the radius of the minimum sphere which can enclose our target. On the other hand, in our two experimental configurations the number of non-redundant measurements is significantly lower. Indeed this number is equal to 15500 and 600 in Configuration $A$ and $B$ respectively, which corresponds to $0.4 \%$ and $0.02 \%$ of the number of degrees of freedom of the scattered field. To describe and analyze the information content, we have constructed the Ewald sphere in far field (Lipson \& Cochran 1966), (Born \& 
Wolf 1999). The Ewald sphere corresponds to the location $\mathbf{K}=\mathbf{k}_{\mathbf{s}}-\mathbf{k}_{\mathbf{i}}$ in the spectral domain, where $\mathbf{k}_{\mathbf{i}}$ denotes the incident wave vector and $\mathbf{k}_{\mathbf{s}}$ the scattered wave vector. If the transmitter and the receiver could be placed everywhere on a sphere all around the target, all the information in the far field would be reached for a given frequency and the Ewald sphere of radius $2 k_{o}$ would be totally filled. Unfortunately, this cannot be achieved in practical situations.

The accessible portion of the Ewald sphere in Configuration $A$ is plotted in Fig. 5. Even if the information content is low compared to the number of degrees of freedom of the scattered field, the Ewald sphere seems to be relatively well-filled at a first sight. The spectral information is especially truncated along $k_{z}$-axis.

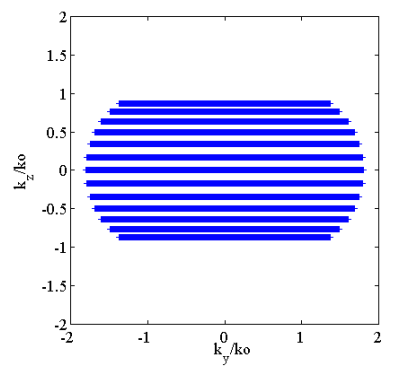

(a) $\left(k_{y} 0 k_{z}\right)$-plane

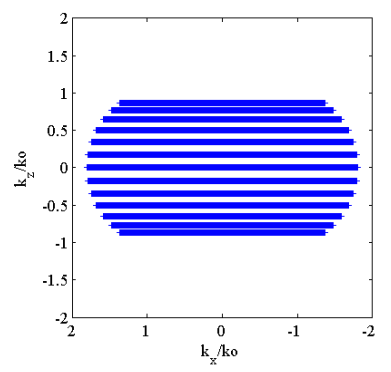

(b) $\left(k_{x} 0 k_{z}\right)$-plane

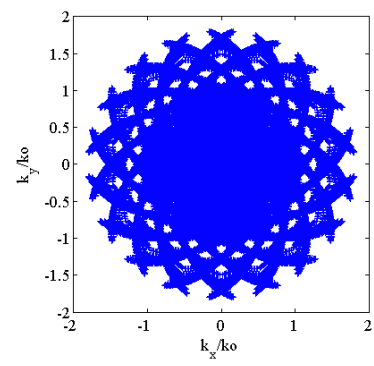

(c) $\left(k_{x} 0 k_{y}\right)$-plane

Figure 5: Ewald sphere corresponding to the measurements in Configuration A.

Fig. 6 shows the accessible portion of the Ewald sphere in Configuration $B$. In this case, it is immediately visible that the Ewald sphere filling is very poor in this case. Indeed, no spectral information along the $k_{z}$-axis is accessible and in the $\left(k_{x} 0 k_{y}\right)$-plane, which is coherent with the fact that the 
source and the receiver are staying in the same plane. Furthermore, many parts of the disk are empty.

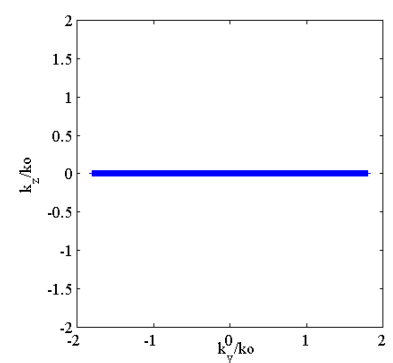

(a) $\left(k_{y} 0 k_{z}\right)$-plane

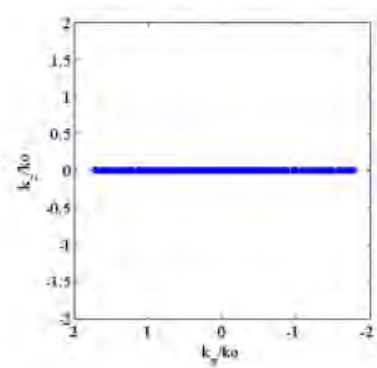

(b) $\left(k_{x} 0 k_{z}\right)$-plane

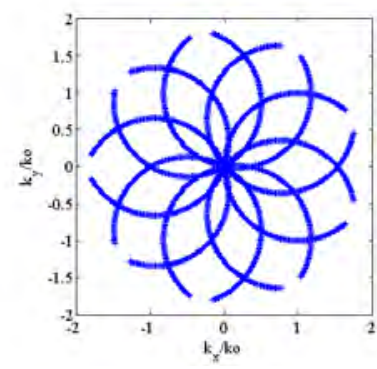

(c) $\left(k_{x} 0 k_{y}\right)$-plane

Figure 6: Ewald sphere corresponding to the measurements in Configuration B.

\section{Vectorial diffraction tomography algorithm}

To reconstruct a 3D image from the scattered field, we chose to work with a qualitative imaging procedure, i.e., the positions and the shapes of the structure can be reconstructed, but the quantitative value of the permittivity can not be reached. This kind of algorithms has two main advantages in the present study: the memory requirement is low (it is thus possible to reconstruct a large domain) and these procedures are generally robust against 
most of the disturbances. In this paper, the induced current maps have been obtained with a vectorial diffraction algorithm (Eyraud et al. 2013).

The field scattered by a target, enclosed in a domain $\Omega$ and illuminated with a monochromatic electromagnetic wave, is calculated in a receiving zone $\Gamma$, at each receiver position $\mathbf{r}^{\mathbf{R}} \in \Gamma$, by means of the observation equation which can be written as

$$
\mathbf{E}^{\mathbf{S}}\left(\mathbf{r}^{\mathbf{R}}\right)=\int_{\Omega} \mathbf{G}\left(\mathbf{r}^{\mathbf{R}}, \mathbf{r}^{\prime}\right) \chi\left(\mathbf{r}^{\prime}\right) \mathbf{E}\left(\mathbf{r}^{\prime}\right) d \mathbf{r}^{\prime}
$$

In a free space configuration, $\mathbf{G}$ is the dyadic free-space Green function between the object zone $\Omega$ and the receiver zone $\Gamma$. $\mathbf{E}$ is the field inside $\Omega$ and $\chi=k\left(\mathbf{r}^{\prime}\right)^{2}-k_{0}^{2}$ is the contrast (with $k_{0}$ the wavenumber in vacuum and $k\left(\mathbf{r}^{\prime}\right)$ the wavenumber in the $\Omega$ domain).

When the following far-field conditions are realized

$$
k_{0} r^{R} \gg 1, \quad r^{R} \gg r^{\prime}, \quad \frac{k_{0} r^{\prime}}{2 r^{R}} \gg 1, \quad \forall r^{R} \in \Gamma, \forall r^{\prime} \in \Omega
$$

the dyadic free-space Green function in spherical coordinates $\mathbf{G}$ can be approximated by (Mishchenko et al. 2002):

$$
\mathbf{G}\left(\mathbf{r}^{\mathbf{R}}, \mathbf{r}^{\prime}\right) \approx \frac{e^{j \mathbf{k}_{\mathbf{s}} \cdot\left(\mathbf{r}^{\mathbf{R}}-\mathbf{r}^{\prime}\right)}}{4 \pi r}\left[\mathbf{I}-\mathbf{e}_{r} \otimes \mathbf{e}_{r}\right]
$$

where $\mathbf{I}$ the identity operator, $\mathbf{a} \otimes \mathbf{b}$ the tensor product between the vector $\mathbf{a}$ and the vector $\mathbf{b}$ and $\left(\mathbf{e}_{r}, \mathbf{e}_{\theta}, \mathbf{e}_{\phi}\right)$ are the unit vectors of the spherical basis.

We assume that the induced current vector $\mathbf{J}\left(\mathbf{r}^{\prime}\right)=\chi\left(\mathbf{r}^{\prime}\right) \mathbf{E}\left(\mathbf{r}^{\prime}\right)$ is collinear with the polarization state of the incident electric field along $\mathbf{u}_{p}^{T}$. The induced current magnitude $\left|J_{p, q}\right|$ created in a target at the position $\mathbf{r}^{\prime}$ - in a lossless embedding medium - is then linked to the measurement of the scattered field 
on a receiver with a selected polarization $\mathbf{u}_{q}^{R}$ by the following equation :

$$
\left|J_{p, q}\left(\mathbf{r}^{\prime}\right)\right| \approx A \frac{\mid \mathbf{E}^{\mathbf{s}}\left(\widetilde{\left.\mathbf{r}^{\mathbf{R}}\right)} \cdot \mathbf{u}_{q}^{R}(\mathbf{K}) \mid\right.}{\mathbf{u}_{p}^{T} \cdot \mathbf{u}_{q}^{R}}, \quad \text { if } \mathbf{u}_{p}^{T} \cdot \mathbf{u}_{q}^{R} \neq 0
$$

where $A=\left|4 \pi r^{R} e^{-j \mathbf{k}_{\mathbf{s}} \cdot \mathbf{r}^{\mathbf{R}}}\right|$ is a constant term and $\mathbf{E}^{\mathbf{s}} \widetilde{\left(\mathbf{r}^{\mathbf{R}}\right)} \cdot \mathbf{u}_{q}^{R}$ is the $3 \mathrm{D}$ inverse Fourier transform of $\mathbf{E}^{\mathbf{s}}\left(\mathbf{r}^{\mathbf{R}}\right) \cdot \mathbf{u}_{q}^{R}$. More details on this imaging procedure can be found in (Eyraud et al. 2013).

In this study, the polarization state of the incident field $\mathbf{u}_{p}^{T}$ (resp. of the scattered field $\mathbf{u}_{q}^{R}$ ) is collinear with $\mathbf{e}_{\phi}^{\mathbf{T}}$ (resp. to $\mathbf{e}_{\phi}^{\mathbf{R}}$ ) It can be noticed that these two vectors $\left(\mathbf{e}_{\phi}^{T}, \mathbf{e}_{\phi}^{R}\right)$ are not collinear between them - except in the case where both the source and the receiver are in the equatorial plane - and this is taken into account in our vectorial imaging procedure.

\section{Imaging results}

With the present imaging techniques, the maximum size of the imaging domain is directly linked to the chosen sampling in the spectral domain, so that it is possible to reconstruct a large area with a fine spatial sampling. Here, the imaging domain is equal to a cube (respectively square) with a side length of $60 \lambda$ for the configuration A (respectively B). In a complete configuration, the resolution could be $\frac{\lambda}{2}$ (Born \& Wolf 1999), but with the limited number of measurements that is available here, the resolution will be lower.

\subsection{Configuration A}

The 3D reconstructed map $\left(\left|J_{p, q}\left(\mathbf{r}^{\prime}\right)\right|\right)$ using the vectorial diffraction tomography algorithm is plotted in Fig. 7. As it can be seen on these maps, 
the shape and global size of the scale model can be correctly determined (the cube in which the scale model can be enclosed is represented in black). Information on the interior of the scale model is also obtained. Indeed, looking to the maps in the different planes (Fig. 7 (c), (e), (g)), the core can be located at the right position in the three planes (the estimated position and size of the core - based on our target knowledge - are represented by a black circle on the figures).

\subsection{Configuration $B$}

Let us turn our attention to the configuration with a very low number of measured points. The imaging results in this configuration are plotted in Fig. 8. Due to the geometry of this configuration (Fig. 6), it is not possible to obtain information along the $z$-axis, the reconstructed map is thus two-dimensional (in the (x0y)-plane). Even in this configuration, the overall size of the reconstructed target is in good agreement with its real size (the square in which the scale model can be enclosed in represented in black). The estimated thickness of the mantle (respectively ice layer) is represented by a green (respectively blue) line in Fig. 8. It can be also noticed that a core is still visible on the map and that its size is consistent with the core size of the real object (represented by a black circle on the figures). This reconstruction can be considered as being very encouraging regarding space mission scenarios because it shows that even with a configuration with few measurements, it is still possible to reach the target structure. 


\section{Conclusion}

To extract the structural information on large structures as comets or asteroids, we investigated the possibility to exploit an inverse algorithm especially built to the spatial diversity, such as what can be measured in bistatic configurations. The induced currents have been reconstructed with a vectorial tomography algorithm which takes into account the polarization states of the transmitting and receiving antennas. The advantage of this method is to have a low-memory requirement (in comparison with iterative quantitative inverse methods) and to be robust against disturbances. On the other hand, only the structural information can be retrieved (not the quantitative values

of the permittivities) and the spatial resolution is limited (to $\frac{\lambda}{2}$ in the best case).

A scale model based on the predicted layer pile model was used in this study and we took the advantage of the microwave analogy to reduce the dimensions of the object. As in real conditions few measurements can be acquired, we have tested different configurations, one corresponding to a very critical case in terms of measurement point count. In each case, an analysis of the information content associated with the spectral information was proposed. The results showed that even in the case of very few measurement points, the structure of the scale comet can be characterized and in particular, the presence of a core can be detected.

In this paper, we used a single frequency to reconstruct the target, the use of several frequencies can be a good way to add information. In this case, the reconstructed map can be obtained in a similar way at each frequency and then the common components of all these maps can be found to reach 
the final map.

In future work, it would be interesting to test such imaging procedure on real space scenarios as the one of CONSERT instrument which has sounded the interior of Comet $67 \mathrm{P} /$ Churyumov-Gerasimenko and with real space data. Future work will adapt quantitative imaging procedures to imaging a large target and few measurement points.

\section{Acknowledgement}

The authors acknowledge the opportunity provided by the Centre Commun de Ressources en Microonde to use its fully equipped anechoic chamber.

\section{References}

Asphaug, E., Barucci, A., Belton, M. \& et al. (2010), Deep interior radar imaging of comets, in '41st Lunar and Planetary Science Conference', The Woodlands, Texas, USA, p. Abstract 2670.

Barriot, J.-P., Kofman, W., Herique, A., Leblanc, S. \& Portal, A. (1999), 'A two dimensional simulation of the CONSERT experiment (radio tomography of comet Wirtanen)', Adv. Space Res. vol. 24, 1127 - 1138.

Belton, M., Thomas, P., Veverka, J., Schultz, P., Hearn, M., Feaga, L., Farnham, T., Groussin, O., Li, J.-Y., Lisse, C., McFadden, L., Sunshinea, J., Meech, K., Delamere, W. \& Kissel, J. (2007), 'The internal structure of Jupiter family cometary nuclei from deep impact observations: The "talps" or "layered pile" model', Icarus vol. 191, 573-585. 
Born, M. \& Wolf, E. (1999), Principles of Optics: Electromagnetic Theory of Propagation, Interference and Diffraction of Light, Cambridge University Press, Cambridge, 7th edition.

Bottke-Jr., W. F., Cellino, A., Paolicchi, P. \& Binzel, R. P. (2002), An Overview of the Asteroids: The Asteroids III Perspective, University of Arizona Press, Tucson.

Bucci, O. M. \& Isernia, T. (1997), 'Electromagnetic inverse scattering: retrievable information and measurement strategies', Radio Science vol. 32, 2123-2137.

Ciarletti, V., Levasseur-regourd, A., Lasue, J., Statz, C., Plettemeier, D., Hérique, A., Rogez, Y. \& Kofman, W. (2015), 'CONSERT suggests a change in local properties of $67 \mathrm{P} /$ Churyumov-Gerasimenko's nucleus at depth', Astronomy and Astrophysics vol. 583, A40 1-7.

Davidsson, B., Sierks, H., Gttler, C., Marzari, F., Pajola, M., Rickman, H., AHearn, M. F., Auger, A.-T., El-Maarry, M. R., Fornasier, S., Gutirrez, P. J., Keller, H. U.and Massironi, M., Snodgrass, C., Vincent, J.-B., Barbieri, C., Lamy, P. L., Rodrigo, R., Koschny, D., Barucci, M. A., Bertaux, J.-L., Bertini, I., Cremonese, G., Da Deppo, V., Debei, S., De Cecco, M., Feller, C., Fulle, M., Groussin, O., Hviid, S. F., Hfner, S., Ip, W.-H., Jorda, L., Knollenberg, J., Kovacs, G., Kramm, J.-R., Khrt, E., Kppers, M., La Forgia, F., Lara, L. M., Lazzarin, M., Lopez Moreno, J. J., MoisslFraund, R., Mottola, S., Naletto, G., Oklay, N., Thomas, N. \& Tubiana, C. (2016), 'The primordial nucleus of comet 67P/Churyumov-Gerasimenko', Astronomy and Astrophysics vol. 592(A63). 
Devaney, A. (2012), Mathematical Foundations of Imaging, Tomography and Wavefield Inversion, Cambridge University, Cambridge.

Donn, B. (1990), 'Formation and structure of fluffy cometary nuclei from random accumulation of grains', Astronomy and Astrophysics vol. 235, 441446.

Eyraud, C., Geffrin, J.-M., Litman, A., Sabouroux, P. \& Giovannini, H. (2006), 'Drift correction for scattering measurements', Applied Physics Letters vol. 89, 244104.

Eyraud, C., Geffrin, J.-M., Litman, A. \& Tortel, H. (2015), 'Complex permittivity determination from far-field scattering patterns', IEEE Antennas and Wireless Propagation Letters vol. 14, 309 - 312.

Eyraud, C., Geffrin, J.-M., Sabouroux, P., Chaumet, P., Tortel, H., Giovannini, H. \& A.Litman (2008), 'Validation of a 3D bistatic microwave scattering measurement setup', Radio Science vol. 43, RS4018.

Eyraud, C., Vaillon, R., Litman, A., Geffrin, J.-M. \& Merchiers, O. (2013), 'Polarization effects in 3D vectorial-induced current reconstructions', Journal of Optical Society of America A vol. 30(10), 1967-1974.

Festou, M. C., Keller, H. U. \& Weaver, H. A. (2004), A Brief Conceptual History of Cometary Science, Asteroids II, University of Arizona Press, Tucson.

Geffrin, J.-M., Eyraud, C., Litman, A. \& Sabouroux, P. (2009), 'Optimization of a bistatic microwave scattering measurement setup: From high to low scattering targets', Radio Science vol. 44, RS003837. 
Geffrin, J.-M. \& Sabouroux, P. (2009), 'Fresnel database continuation: Experimental setup and improvements for 3D scattering measurements', Inverse Problems vol. 25, 024001.

Gombosi, T. \& Houpis, H. (1986), 'An icy-glue model of cometary nuclei', Nature vol. 324, 43-44.

Grimm, R., Stillman, D., Sava, P. \& Ittharat, D. (2015), 'Radio reflection imaging of asteroid and comet interiors II: Results and recommendations', Advances in Space Research vol. 55, 2166-2176.

Gurg, G. \& Wolf, E. (2001), 'Relation between computed tomography and diffraction tomography', Journal of Optical Society of America A vol. 18, 2132-2137.

Gustafson, B. (2009), Scaled analog experiments in slectromagnetic scattering, In A.A. Kokhanovsky Light Scattering Review 4, pp. 3 - 30.

Heggy, E., Palmer, E., Clifford, S., Righter, K. \& Hérique, A. (2012), 'Radar properties of comets: Parametric dielectric modeling of comet 67P/Churyumov-Gerasimenko', Icarus vol. 221, 925-939.

Hegler, S., Statz, C., Hahnel, R., Plettemeier, D., Hérique, A. \& Kofman, W. (2013), 'Operation of CONSERT aboard Rosetta during the descent of Philae', Planetary and Space Science vol. 89, 151-158.

Herique, A., Agnus, B., Asphaug, E. \& et al. (n.d.), 'Direct observations of asteroid interior and regolith structure: Science measurement requirements', Advances in Space Research submitted in the same issue. 
Herique, A., Kofman, W., Beck, P., Bonal, L., Buttarazzi, I., Heggy, E., Lasue, J., Levasseur-Regourd, A. C., Quirico, E. \& Zine, S. (2017), 'Cosmochemical implications of CONSERT permittivity characterization of 67P/CG', Monthly Notices of the Royal Astronomicla Society vol. 462 (Suppl. 1) - S516S532.

Herique, A., Kofman, W., Hagfors, T., Caudal, G. \& Ayanides, J.-P. (1999), 'A characterization of a comet nucleus interior: inversion of simulated radio frequency data', Planetary and Space Science vol. 47, 774-893.

Kofman, W., Barbin, Y., Klinger, J., Levasseur-Regourd, A.-C., Barriot, J.-P., Herique, A., Hagfors, T., Nielsen, E. \& Gr, E. (1998), 'The Comet Nucleus Sounding Experiment by Radio-wave Transmission (CONSERT)', Advances in Space Research vol. 21, 1589-1598.

Kofman, W., Herique, A., Barbin, Y., Barriot, J.-P., Ciarletti, V., Clifford, S., Edenhofer, P., Elachi, C., Eyraud, C., Goutail, J.-P., Heggy, E., Jorda, L., Lasue, J., Levasseur-Regourd, A.-C., Nielsen, E., Pasquero, P., Preusker, F., Puget, P., Plettemeier, D., Rogez, Y., Sierks, H., Statz, C., Svedhem, H., Williams, I., Zine, S. \& Van Zyl, J. (2015), 'Properties of the $67 \mathrm{P} /$ Churyumov-Gerasimenko interior revealed by CONSERT radar', Science vol. 349(issue 6247), 0639-1 - 0639-6.

Kofman, W., Herique, A., Goutail, J.-P., Hagfors, T., Williams, I., Nielsen, E., Barriot, J.-P., Barbin, Y., Elachi, C., Edenhofer, P., LevasseurRegourd, A.-C., Plettemeier, D., Picardi, G., Seu, R. \& Svedhem, V. (2007), 'The Comet Nucleus Sounding Experiment by Radiowave Trans- 
mission (CONSERT): A short description of the instrument and of the commissioning stages.', Space Science Reviews vol. 128(413-432).

Kofman, W. \& Safaeinili, A. (2004), Peering inside near-Earth objects with radio tomography, Cambridge University Press, Cambridge.

Lipson, H. \& Cochran, W. (1966), The Determination of Crystal Structure, G.Bell And Sons Limited, Bell, London.

Litman, A. \& Crocco, L. (2009), 'Guest editor introduction', Inverse Problems vol. 26, 020201.

Mishchenko, M., Travis, L. \& Lacis, A. (2002), Scattering, Absorption, and Emission of Light by Small Particles, Cambridge University Press, Cambridge, http://www.giss.nasa.gov/staff/mmishchenko/books.html.

Pursiainen, S. \& Kaasalainen, M. (2014), 'Detection of anomalies in radio tomography of asteroids: Source count and forward errors', Planetary and Space Science pp. 36-47.

Pursiainen, S. \& Kaasalainen, M. (2015), 'Electromagnetic 3d subsurface imaging with source sparsity for a synthetic object', Inverse Problems vol. 31(issue 12), 125004(1-17).

Sabouroux, P. \& Ba, D. (2011), 'Epsimu, a tool for dielectric properties measurement of porous media: application in wet granular materials characterization.', PIER B vol. 29, 191-207.

Safaeinili, A., Gulkis, S., Hofstadter, M. \& Jordan, R. (2002), 'Probing the 
interior of asteroids and comets using radio reflection tomography', Meteoritics and Planetary Science vol. 37, 1953-1963.

Sava, P., Ittharat, D., Grimm, R. \& Stillman, D. (2015), 'Radio reflection imaging of asteroid and comet interiors I: Acquisition and imaging theory', Advances in Space Research vol. 55, 2149-2165.

Sunshine, J., Thomas, N., El-Maarry, M. \& Farnham, T. L. (2016), 'Evidence for geologic processes on comets', J. Geophys. Res. Planets, vol. 121, 2194-2210.

Virkki, A. \& Muinonen, K. (2016), 'Radar scattering by planetary surfaces modeled with laboratory-characterized particles', Icarus vol. 269, 38-49.

Weissman, P. (1986), 'Are cometary nuclei primordial rubble piles?', Nature vol. 320, 242-244. 


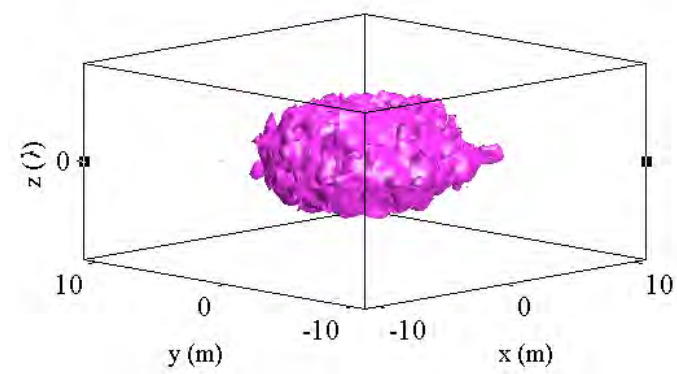

(a)

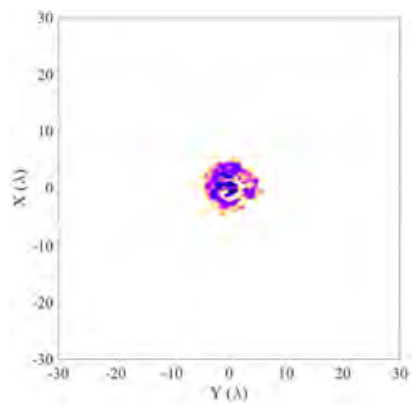

(b) (x0y) plane

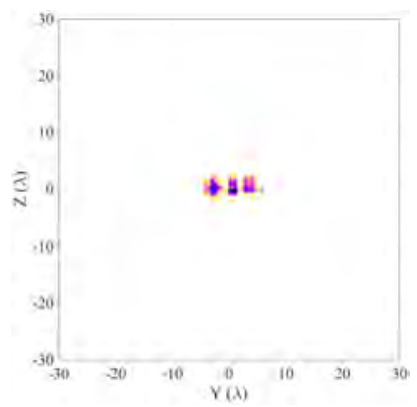

(d) $(\mathrm{y} 0 \mathrm{z})$ plane

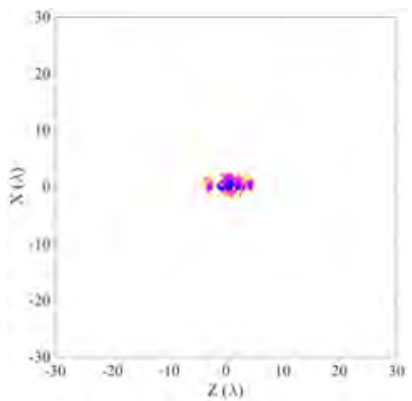

(f) $(\mathrm{x} 0 \mathrm{z})$ plane

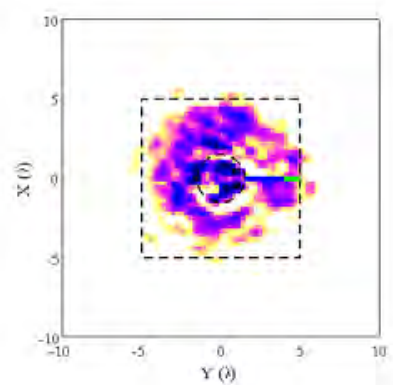

(c) (x0y) plane

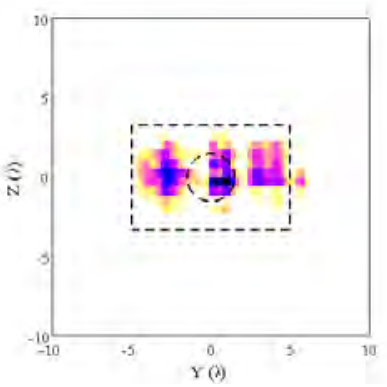

(e) $(\mathrm{y} 0 \mathrm{z})$ plane

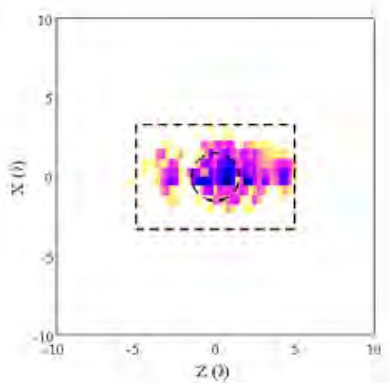

$25 \quad(\mathrm{~g})(\mathrm{x} 0 \mathrm{z})$ plane

Figure 7: Qualitative reconstructed images: (a) 3D view of the reconstruction, (b) (and zoom in (c)) imaging map in the $(x 0 y)$ plane, (d) (and zoom in (e)) imaging map in the $(y 0 z)$ plane, (f) (and zoom in $(\mathrm{g})$ ) imaging map in the $(x 0 z)$ plane. The box in which the scale model can be enclosed in denoted in black. The estimated position and size of the 


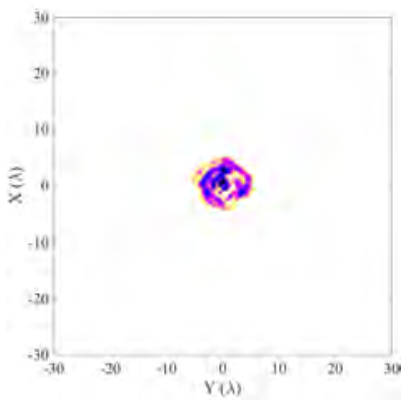

(a)

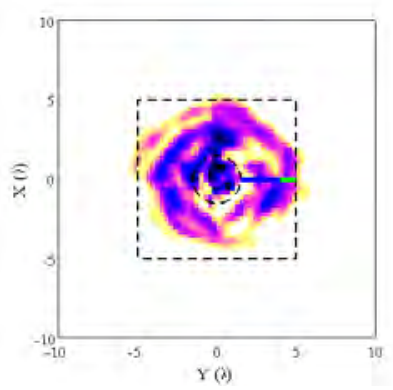

(b)

Figure 8: Qualitative reconstructed of our scaled comet model from experimental fields. The box in which the scale model can be enclosed is represented in black. The estimated thickness of the mantle (respectively ice layer) is represented by a green (respectively blue) line and the estimated position and size of the core are represented by a black circle. The image (b) is a zoom of the image (a). 